\title{
Nonequilibrium thermodynamics and phase transition of Ehrenfest urns with interactions
}

\author{
Chi-Ho Cheng $\circledast^{1, *}$ and Pik-Yin Lai $\odot^{2, \dagger}$ \\ ${ }^{1}$ Department of Physics, National Changhua University of Education, Changhua 500, Taiwan \\ ${ }^{2}$ Department of Physics and Center for Complex Systems, National Central University, Taoyuan 320, Taiwan
}

(Received 5 January 2021; accepted 3 May 2021; published 21 May 2021)

\begin{abstract}
Ehrenfest urns with interaction that are connected in a ring is considered as a paradigm model for nonequilibrium thermodynamics and is shown to exhibit two distinct nonequilibrium steady states (NESS) of uniform and nonuniform particle distributions. As the interparticle attraction varies, a first-order nonequilibrium phase transition occurs between these two NESSs characterized by a coexistence regime. The phase boundaries, the NESS particle distributions near saddle points and the associated particle fluxes, average urn population fractions, and the relaxational dynamics to the NESSs are obtained analytically and verified numerically. A generalized nonequilibrium thermodynamics law is also obtained, which explicitly identifies the heat, work, energy, and entropy of the system.
\end{abstract}

DOI: 10.1103/PhysRevResearch.3.023134

\section{INTRODUCTION}

The classic Ehrenfest model [1] was introduced to solve the reversal and Poincare recurrence paradox [2,3]. It describes a total of $N$ particles in two urns that can randomly jump from one urn to the other with equal probability. The system has a Poincare cycle time of $2^{N}$ [4], providing a fundamental link between reversible microscopic dynamics and irreversible thermodynamics.

Later on, a directional jumping rate between urns was introduced [5,6], and extensions to multiurns [7-9] were made. Although there are various modifications [10-21] of the classic Ehrenfest model, or even extensions by incorporating nonlinear contribution [22-25], particles do not interact or the interaction is merely phenomenological. In fact, pairwise particle interaction in the same urn has been considered recently in the two-urn model [26]. The interacting two-urn Ehrenfest model can exhibit phase transitions by varying the interaction strength and directional jumping rate from which the relaxation time and Poincaré cycle can be derived. The multiurn system with interaction and unbiased directional jumping will evolve to equilibrium and has been shown to exhibit different levels of nonuniformity emerge with the coexistence of uniform and nonuniform phases [27]. Contrary to the better understood equilibrium cases, nonequilibrium statistical physics remains challenging, partly due to the lack of well-characterised states. Even for nonequilibrium steady states (NESS), it is difficult to describe nonequilibrium phase transitions between different NESS and their relationship to some microscopic models. For example, selection rules, such

\footnotetext{
*phcch@cc.ncue.edu.tw

†pylai@phy.ncu.edu.tw
}

Published by the American Physical Society under the terms of the Creative Commons Attribution 4.0 International license. Further distribution of this work must maintain attribution to the author(s) and the published article's title, journal citation, and DOI. as maximal or minimal entropy production principles [28-30] have been proposed to determine the nonequilibrium states. Yet, universal guiding principles are still lacking.

In this paper, we consider urns with intra-urn interactions connected in a one-dimensional ring with directional jumping rates. We will show that the system has nonequilibrium steady states in uniform and nonuniform phases that can coexist. For high directional jumping rates with appropriate interaction strengths, the steady states become unstable. The phase diagram will be obtained analytically, and the relaxation dynamics to the NESS will be studied. The relationship between nonequilibrium thermodynamical variables such as the internal entropy production rate, the rate of work done applied to the system, will be shown to obey a generalized thermodynamic law. Finally, we will demonstrate that, in the coexistence region, the internal entropy production rate fails to select the favorable steady state.

\section{EHRENFEST URNS IN A RING}

We consider three urns as illustrated in Fig. 1 as this already captures the nonequilibrium physics. The state of the system is labeled by $\vec{n}=\left(n_{1}, n_{2}, n_{3}\right)$ where $n_{i}$ is the number of particles in the $i$ th urn with $n_{1}+n_{2}+n_{3}=N$, the fixed total particle number. Similar to previous models [26,27], we include a pairwise attractive (repulsive) interaction with negative (positive) energy $J$ for particles in the same urn. Particles in different urns do not interact. A particle in the $i$ th urn (initial state $\vec{n}$ ) jumps to the $j$ th urn (final state $\vec{m}$ ) with corresponding transition probability,

$$
T_{\vec{m}, \vec{n}}=\frac{1}{e^{-\frac{g}{N}\left(n_{i}-n_{j}-1\right)}+1},
$$

where $m_{i}=n_{i}-1$ and $m_{j}=n_{j}+1 . g \equiv N J \beta$ where $\beta$ is the inverse of effective temperature. Without interaction $(g=0)$, we have $T_{\vec{m}, \vec{n}}=\frac{1}{2}$. Next, a jumping rate is introduced such that the probability of anticlockwise (clockwise) direction is $p(q)$. For the sake of convenience, $p+q=1$ is imposed for which only changes the time scale. 


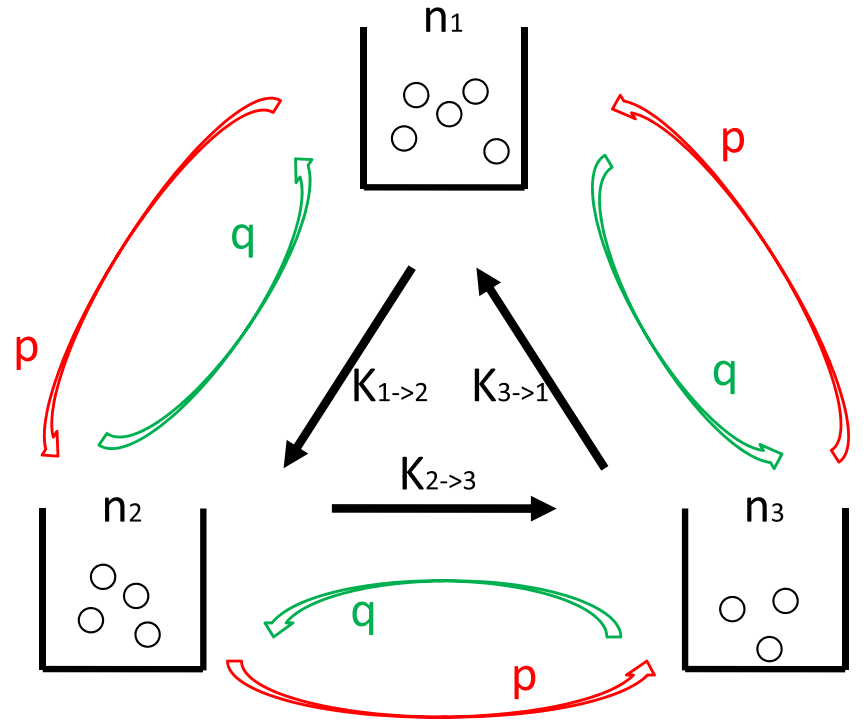

FIG. 1. Schematic diagram of the model. Three urns with particle numbers $n_{1}, n_{2}$, and $n_{3}$ are connected in a ring. The direct jumping rate in anticlockwise (clockwise) direction is $p(q) . K_{i \rightarrow j}$ represents net particle flow rate from the $i$ th to the $j$ th urn.

After $s$ steps from the initial state, the probability of the state $\vec{n}$ is denoted by $\rho(\vec{n}, s)$. The master equation from the $s$ th to the $(s+1)$ th time step can be written as

$$
\rho(\vec{n}, s+1)-\rho(\vec{n}, s)=\sum_{\vec{m}}\left(W_{\vec{n}, \vec{m}} \rho(\vec{m}, s)-W_{\vec{m}, \vec{n}} \rho(\vec{n}, s)\right),
$$

where

$$
W_{\vec{m}, \vec{n}}=\frac{n_{i}}{N} p T_{\vec{m}, \vec{n}},
$$

holds if the particle jumps from the $i$ th to the $j$ th urn is anticlockwise, i.e., $(i, j)=(1,2),(2,3),(3,1)$, and

$$
W_{\vec{m}, \vec{n}}=\frac{n_{i}}{N} q T_{\vec{m}, \vec{n}},
$$

if $(i, j)=(2,1),(1,3),(3,2)$, which represents clockwise jumps.

Finally, the net particle flow rate from the $i$ th to the $j$ th urn (see Fig. 1 for illustration) is given by

$$
K_{i \rightarrow j}(s) \equiv N \sum_{\vec{n}}\left(W_{\vec{m}, \vec{n}}-W_{\vec{m}^{\prime}, \vec{n}}\right) \rho(\vec{n}, s),
$$

where $\quad m_{i}=n_{i}-1, \quad m_{j}=n_{j}+1, \quad$ and $\quad m_{i}^{\prime}=n_{i}+1$, $m_{j}^{\prime}=n_{j}-1$.

\section{EQUILIBRIUM STATES}

If $\lim _{s \rightarrow \infty} \rho(\vec{n}, s)$ exists, it defines the steady state $\rho^{\mathrm{ss}}(\vec{n})$. Taking the limit $s \rightarrow \infty$ in Eq. (2) would lead to an equation in which $\rho^{\text {ss }}(\vec{n})$ satisfies. No closed form exists in general. But for the case of $p=q=\frac{1}{2}$, an analytic expression for $\rho^{\text {ss }}$ can be obtained

$$
\rho^{\mathrm{ss}}(\vec{n})=\frac{N !}{n_{1} ! n_{2} ! n_{3} !} e^{-\frac{g}{2 N}\left(n_{1}^{2}+n_{2}^{2}+n_{3}^{2}\right)},
$$

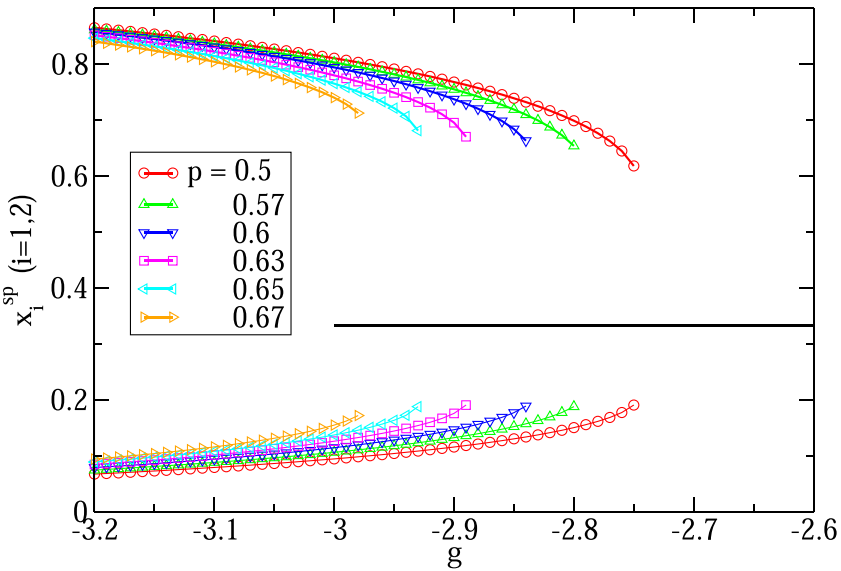

FIG. 2. Occupation fraction at the stable saddle point in the $i$ th urn, $x_{i}^{\mathrm{sp}}$, as a function of coupling constant $g$ for different $p$. Up to the cyclic permutation, we consider $x_{1}^{\text {sp }}$ the largest fraction, and $x_{2}^{\mathrm{sp}}$ is the occupation fraction in the next urn along the $p$ direction. The remaining $x_{3}^{\mathrm{sp}}=1-x_{1}^{\mathrm{sp}}-x_{2}^{\mathrm{sp}}$. Solid line (black) represents the stable saddle point $x_{1}^{\mathrm{sp}}=x_{2}^{\mathrm{sp}}=x_{3}^{\mathrm{sp}}=\frac{1}{3}$ (uniform distribution) shared by all values of $p$.

up to a normalization constant. This steady state is also the equilibrium state, because it satisfies the condition of detailed balance

$$
W_{\vec{m}, \vec{n}} \rho^{\mathrm{ss}}(\vec{n})=W_{\vec{n}, \vec{m}} \rho^{\mathrm{ss}}(\vec{m}),
$$

which can be verified by direct substitution. Results for the general case of $M$ urns at equilibrium can be found in Ref. [27]. For the three urns case here with the constraint $n_{1}+n_{2}+n_{3}=N$, one can define the population fraction with $x_{i} \equiv n_{i} / N$ and rewrite $\rho^{\text {ss }}$ in terms of $\vec{x} \equiv$ $\left(x_{1}, x_{2}\right)$ (two independent variables), $\rho^{\mathrm{ss}}(\vec{x})=\exp \{N f(\vec{x})-$ $\left.\frac{1}{2} \log \left[(2 \pi N)^{2} x_{1} x_{2}\left(1-x_{1}-x_{2}\right)\right]+O\left(N^{-1}\right)\right\}$ in large $N$ limit, where

$$
\begin{aligned}
f(\vec{x})= & -x_{1} \ln x_{1}-x_{2} \ln x_{2} \\
& -\left(1-x_{1}-x_{2}\right) \ln \left(1-x_{1}-x_{2}\right) \\
& -\frac{g}{2}\left(x_{1}^{2}+x_{2}^{2}+\left(1-x_{1}-x_{2}\right)^{2}\right) .
\end{aligned}
$$

The saddle point approximation [31] gives asymptotic form

$$
\rho^{\mathrm{ss}}(\vec{x}) \propto e^{N f\left(\vec{x}^{\mathrm{sp}}\right)+\frac{N}{2} \sum_{i, j} \partial_{i j} f\left(\vec{x}^{\mathrm{sp}}\right)\left(x_{i}-x_{i}^{\mathrm{sp}}\right)\left(x_{j}-x_{j}^{\mathrm{sp}}\right)},
$$

where $\vec{x}^{\mathrm{sp}}$ is the saddle point(s) satisfying $\partial_{1} f=\partial_{2} f=0$, $\partial_{11} f<0$, and $\partial_{11} f \partial_{22} f-\left(\partial_{12} f\right)^{2}>0$. This condition leads to

$$
x_{1}^{\mathrm{sp}} e^{g x_{1}^{\mathrm{sp}}}=x_{2}^{\mathrm{sp}} e^{g x_{2}^{\mathrm{sp}}}=x_{3}^{\mathrm{sp}} e^{g x_{3}^{\mathrm{sp}}} .
$$

The solutions at different coupling constant $g$ are shown in the data of $p=0.5$ in Fig. 2. The steady net particle flow at equilibrium from Eq. (5) reads

$$
\frac{K_{i \rightarrow j}^{\mathrm{ss}}}{N}=\frac{x_{i}^{\mathrm{sp}} e^{g x_{i}^{\mathrm{sp}}}-x_{j}^{\mathrm{sp}} e^{g x_{j}^{\mathrm{sp}}}}{2\left(e^{g x_{i}^{\mathrm{sp}}}+e^{g x_{j}^{\mathrm{sp}}}\right)}+O(1 / N),
$$

which gives $K_{1 \rightarrow 2}^{\mathrm{ss}}=K_{2 \rightarrow 3}^{\mathrm{ss}}=K_{3 \rightarrow 1}^{\mathrm{ss}}=0$ from Eq. (10), in large $N$ limit. At equilibrium, there is no net particle flow between any two urns. In addition, it is also easy to see from 
Eqs. (5) and (7) that for $p=q=\frac{1}{2}$, all fluxes $K_{i \rightarrow j}^{\mathrm{ss}}=0$ at equilibrium. On the other hand, or the nonequilibrium case of $p \neq q$, there can be nonvanishing circulating fluxes as in other general NESS systems [32-34].

\section{UNIFORM AND NONUNIFORM NONEQUILIBRIUM STEADY STATES}

So far, the recurrence relation in Eq. (2) cannot be solved analytically even for NESS. In this section, we will transform Eq. (2) into the Fokker-Planck equation. Let the (physical) time $t=\frac{\tau_{1}}{N} s$, where $\tau_{1}$ is the time scale of each single step from $s$ to $s+1$. Replace $\rho(\vec{n}, s+1)-\rho(\vec{n}, s)$ by $\rho(\vec{n}, t+$ $\left.\frac{\tau_{1}}{N}\right)-\rho(\vec{n}, t)=\frac{\tau_{1}}{N} \frac{\partial \rho}{\partial t}+O\left(\left(\frac{\tau_{1}}{N}\right)^{2}\right)$. Equation (2) can be rewritten as

$$
\frac{\tau_{1}}{N} \frac{\partial \rho(\vec{n}, t)}{\partial t}=\sum_{\vec{m}}\left(W_{\vec{n}, \vec{m}} \rho(\vec{m}, t)-W_{\vec{m}, \vec{n}} \rho(\vec{n}, t)\right) .
$$

Substituting Eqs. (1), (3)-(4) into Eq. (12) gives

$$
\begin{aligned}
& \frac{\tau_{1}}{N} \frac{\partial \rho(\vec{x}, t)}{\partial t} \\
& =p \sum_{k=1}^{\infty} \frac{1}{k ! N^{k}}\left(\frac{\partial}{\partial x_{1}}-\frac{\partial}{\partial x_{2}}\right)^{k}\left[\frac{x_{1}}{e^{-g\left(x_{1}-x_{2}\right)}+1} \rho(\vec{x}, t)\right] \\
& \quad+q \sum_{k=1}^{\infty} \frac{1}{k ! N^{k}}\left(-\frac{\partial}{\partial x_{1}}+\frac{\partial}{\partial x_{2}}\right)^{k}\left[\frac{x_{2}}{e^{g\left(x_{1}-x_{2}\right)}+1} \rho(\vec{x}, t)\right] \\
& \quad+[\text { cyclic terms }],
\end{aligned}
$$

which is known as the Kramers-Moyal expansion [35,36]. From now on, we take $\tau_{1}=1$ for convenience.

If we further keep terms up to $O\left(1 / N^{2}\right)$, Eq.(13) becomes the Fokker-Planck equation

$$
\begin{aligned}
\frac{\partial \rho(\vec{x}, t)}{\partial t}= & -\sum_{i=1}^{2} \frac{\partial}{\partial x_{i}}\left[A_{i}(\vec{x}) \rho(\vec{x} ; t)\right] \\
& +\frac{1}{2 N} \sum_{i, j=1}^{2} \frac{\partial^{2}}{\partial x_{i} \partial x_{j}}\left[B_{i j}(\vec{x}) \rho(\vec{x} ; t)\right],
\end{aligned}
$$

where

$$
\begin{aligned}
A_{1}(\vec{x})= & -\frac{p x_{1}}{e^{-g\left(x_{1}-x_{2}\right)}+1}+\frac{q x_{2}}{e^{g\left(x_{1}-x_{2}\right)}+1} \\
& -\frac{q x_{1}}{e^{-g\left(2 x_{1}+x_{2}-1\right)}+1}+\frac{p\left(1-x_{1}-x_{2}\right)}{e^{g\left(2 x_{1}+x_{2}-1\right)}+1}, \\
A_{2}(\vec{x})= & -\frac{q x_{2}}{e^{-g\left(x_{2}-x_{1}\right)}+1}+\frac{p x_{1}}{e^{g\left(x_{2}-x_{1}\right)}+1} \\
& -\frac{p x_{2}}{e^{-g\left(2 x_{2}+x_{1}-1\right)}+1}+\frac{q\left(1-x_{1}-x_{2}\right)}{e^{g\left(2 x_{2}+x_{1}-1\right)}+1}, \\
B_{11}(\vec{x})= & \frac{p x_{1}}{e^{-g\left(x_{1}-x_{2}\right)+1}}+\frac{q x_{2}}{e^{g\left(x_{1}-x_{2}\right)}+1} \\
& +\frac{q x_{1}}{e^{-g\left(2 x_{1}+x_{2}-1\right)}+1}+\frac{p\left(1-x_{1}-x_{2}\right)}{e^{g\left(2 x_{1}+x_{2}-1\right)}+1},
\end{aligned}
$$

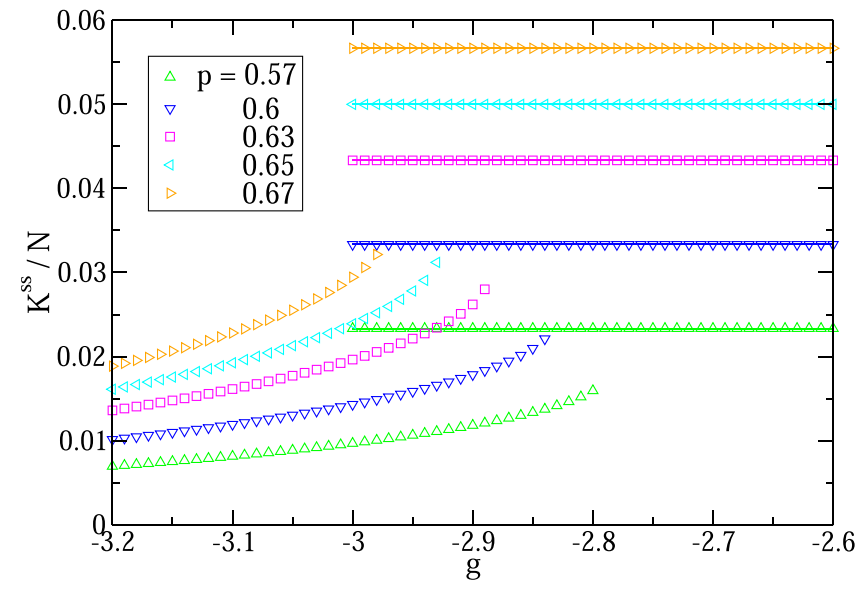

FIG. 3. Net particle flow of the uniform and nonuniform NESS as a function of coupling constant $g$ for different $p$. Symbols with and without lines represent uniform and nonuniform distributions, respectively.

$$
\begin{array}{r}
B_{22}(\vec{x})=\frac{q x_{2}}{e^{-g\left(x_{2}-x_{1}\right)}+1}+\frac{p x_{1}}{e^{g\left(x_{2}-x_{1}\right)}+1} \\
+\frac{p x_{2}}{e^{-g\left(2 x_{2}+x_{1}-1\right)}+1}+\frac{q\left(1-x_{1}-x_{2}\right)}{e^{g\left(2 x_{2}+x_{1}-1\right)}+1}, \\
B_{12}(\vec{x})=B_{21}(\vec{x})=-\frac{p x_{1}}{e^{-g\left(x_{1}-x_{2}\right)}+1}-\frac{q x_{2}}{e^{g\left(x_{1}-x_{2}\right)}+1} .
\end{array}
$$

The WKB approximation [37-41] yields the saddle points [31] $\vec{x}^{\mathrm{sp}}=\left(x_{1}^{\mathrm{sp}}, x_{2}^{\mathrm{sp}}\right)$ as

$$
A_{1}\left(\vec{x}^{\mathrm{sp}}\right)=0, \quad A_{2}\left(\vec{x}^{\mathrm{sp}}\right)=0,
$$

whose solutions at different $g$ and $p$ are shown in Fig. 2. The physical meaning of Eq. (20) is that $K_{1 \rightarrow 2}^{\mathrm{ss}}=K_{2 \rightarrow 3}^{\mathrm{ss}}=K_{3 \rightarrow 1}^{\mathrm{ss}}=$ $K^{\text {ss }}$, i.e., a constant nonzero cyclic flux of net particle along the ring which can be calculated from Eq. (5) as

$$
\frac{K^{\mathrm{ss}}}{N}=\frac{p x_{1}^{\mathrm{sp}} e^{g x_{1}^{\mathrm{sp}}}-q x_{2}^{\mathrm{sp}} e^{g x_{2}^{\mathrm{sp}}}}{e^{g x_{1}^{\mathrm{sp}}}+e^{g x_{2}^{\mathrm{sp}}}} .
$$

For uniform NESS $\left(x_{1}=x_{2}=\frac{1}{3}\right)$, one obtains $K_{\mathrm{u}}^{\mathrm{ss}}=$ $\frac{N}{6}(p-q)$ and, for nonuniform NESS, $K_{\mathrm{nu}}^{\mathrm{ss}}$ can be computed using the nonuniform saddle point from Eqs. (20). The $K_{\mathrm{u}}^{\mathrm{ss}}$ and $K_{\mathrm{nu}}^{\mathrm{ss}}$ NESS fluxes as a function of $g$ at different $p$ are shown in Fig. 3 indicating that the particle flux of the uniform NESS is always significantly larger than that of the nonuniform NESS. Notice that there is a coexistence region of uniform and nonuniform saddle points.

The NESS can be further analysed by expanding around the saddle point, i.e., using $A_{i}(\vec{x}) \simeq \sum_{j} \partial_{j} A_{i}\left(\vec{x}^{\mathrm{sp}}\right)\left(x_{j}-x_{j}^{\mathrm{sp}}\right) \equiv$ $\sum_{j} a_{i j}\left(x_{j}-x_{j}^{\mathrm{sp}}\right)$ and $B_{i j}(\vec{x}) \simeq B_{i j}\left(\vec{x}^{\mathrm{sp}}\right)=b_{i j}$, the steady-state particle distribution is

$$
\rho^{\mathrm{ss}}(\vec{x}) \propto \exp \left[N \sum_{i, j=1}^{2} c_{i j}\left(x_{i}-x_{i}^{\mathrm{sp}}\right)\left(x_{j}-x_{j}^{\mathrm{sp}}\right)\right],
$$

where the matrix $\mathbf{c}$ is uniquely determined by the Lyapunov equations $\mathbf{a} \mathbf{c}^{-1}+\mathbf{c}^{-1} \mathbf{a}^{\mathrm{t}}=2 \mathbf{b}$ (see Appendix A for details). The detailed balance condition can be transformed into $\mathbf{c}=$ $\mathbf{b}^{-1} \mathbf{a}$ (see Appendix B for details).

Obviously $x_{1}^{\mathrm{sp}}=x_{2}^{\mathrm{sp}}=x_{3}^{\mathrm{sp}}=\frac{1}{3}$ is always a saddle point of uniform population fraction. At this uniform NESS, 


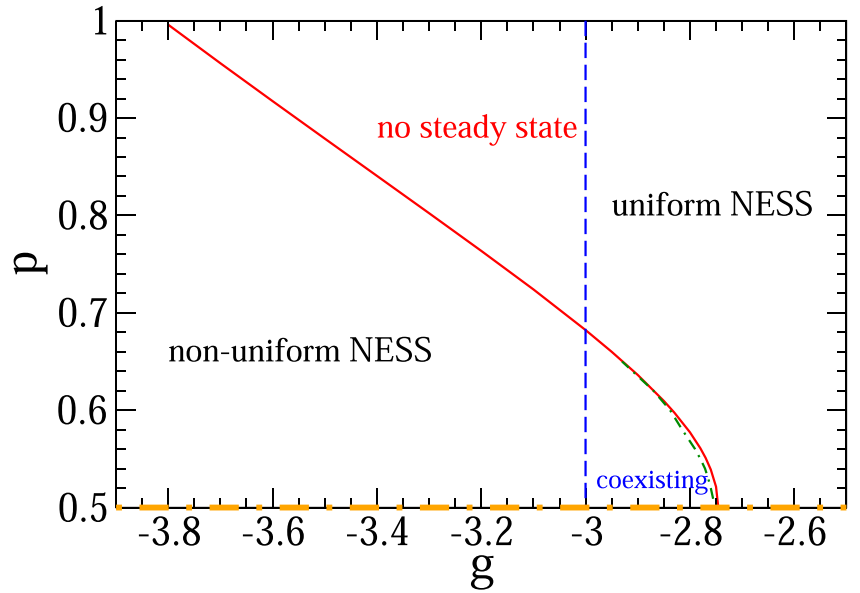

FIG. 4. Phase diagram of the interacting Ehrenfest model of three urns connected in a ring. There are four regions, which represent uniform NESS (particles uniformly distributed in three urns), nonuniform NESS, coexistence (both uniform and nonuniform NESSs are stable), and no steady state. The stability boundaries of the uniform NESS and nonuniform NESS are denoted by the vertical dashed line and solid curve respectively. The first-order phase transition boundary in the coexistence regime is denoted by the dashed-dotted curve (see Fig. 11 in Appendix C for a magnification).

we have

$$
\begin{gathered}
\mathbf{a}=-\frac{1}{2}\left(\begin{array}{cc}
1+p+\frac{g}{2} & p-q \\
q-p & 1+q+\frac{g}{2}
\end{array}\right), \\
\mathbf{b}=\frac{1}{6}\left(\begin{array}{cc}
2 & -1 \\
-1 & 2
\end{array}\right)
\end{gathered}
$$

which gives

$$
\mathbf{c}=-\frac{g+3}{2}\left(\begin{array}{ll}
2 & 1 \\
1 & 2
\end{array}\right)
$$

Hence its stability requires $g>-3$. The stable region for the nonuniform phase can be determined analytically in a similar manner and the results agree with the analytic ones of the phase boundary in Fig. 4. In fact, the nonequilibrium physics of the system can be summarized by the phase diagram in Fig. 4 whose phase boundaries can be determined analytically (see Appendix $\mathrm{C}$ for derivations). As the particle interaction is strongly repulsive (positively large $g$ ), the particles are uniformly distributed in every urn. On the other hand, the particles "prefer" to stay in the same urn (nonuniform distribution) if they are strongly attractive (negatively large $g$ ). In between, for low jumping rate, $1-p_{\mathrm{s}}<p<p_{\mathrm{s}}$ ( $p_{\mathrm{s}} \simeq 0.6823$ ), there is a coexistence region where both uniform and nonuniform distribution are locally stable. There is a first-order nonequilibrium phase transition between the uniform and nonuniform NESSs whose transition value of $g$ can be determined from the analytic result of $K_{\mathrm{u}}^{\mathrm{ss}}$ and $K_{\mathrm{nu}}^{\mathrm{ss}}$ (see Appendix $\mathrm{C}$ for details) together with the results of mean steady-state flux. The latter has to be determined numerically using (2). The first-order phase transition line (dashed-dotted curve) is also shown in Fig. 4. It is close to the stability line of the nonuniform NESS (for magnification, see Fig. 11 in Appendix C). As the jumping rate becomes higher, i.e., $p>p_{\mathrm{s}}$

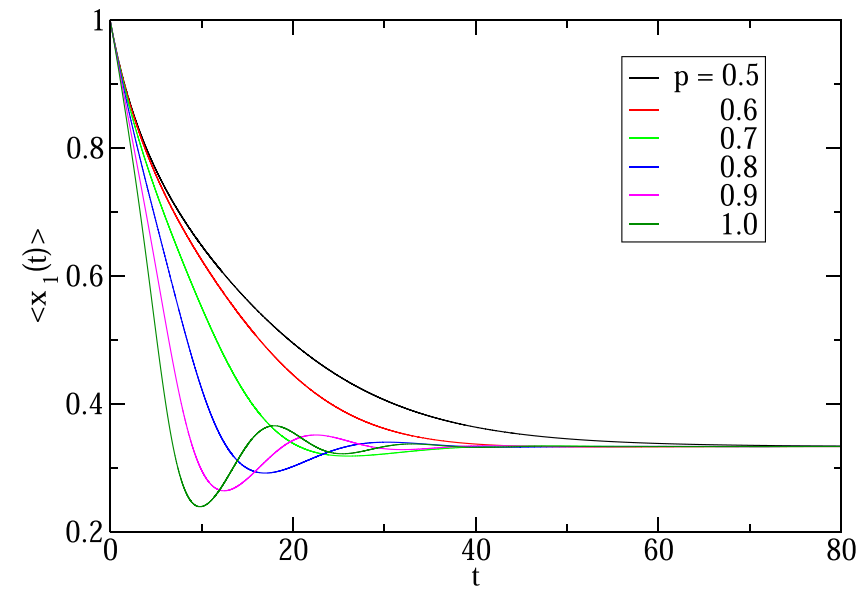

FIG. 5. $\left\langle x_{1}(t)\right\rangle$ as a function of $t$ at different $p$ with $g=-2.5$. The initial state is $\vec{x}(0)=(1,0,0)$ and the steady state is uniform. The result is numerically solved from Eq. (2) for $N=300$.

(or $p<1-p_{\mathrm{s}}$ ), the system is far from equilibrium and steady states do no longer exist.

\section{RELAXATION TO STEADY STATES}

In this section, we studied how the system evolves to its steady states. When the system is initially away from its steady state, it will relax (thermalized) towards the nearby stable NESS whose dynamics near the NESS is determined by the eigenvalues of a. From Eq. (14), and expand around the saddle point $\vec{x}^{\text {sp }}$, we get the evolution of the average of particle numbers in the first and second urn as

$$
\frac{d}{d t}\left(\begin{array}{c}
\left\langle x_{1}(t)\right\rangle \\
\left\langle x_{2}(t)\right\rangle
\end{array}\right)=\mathbf{a}\left(\begin{array}{c}
\left\langle x_{1}(t)\right\rangle-x_{1}^{\mathrm{sp}} \\
\left\langle x_{2}(t)\right\rangle-x_{2}^{\mathrm{sp}}
\end{array}\right) .
$$

At uniform phase, $x_{1}^{\mathrm{sp}}=x_{2}^{\mathrm{sp}}=x_{3}^{\mathrm{sp}}=\frac{1}{3}$, its solution is

$$
\begin{aligned}
\left\langle x_{1}(t)\right\rangle= & \frac{1}{3}+\left\{\left(x_{1}(0)-\frac{1}{3}\right) \cos \left(\frac{2 \pi t}{\tau_{\mathrm{osc}}}\right)\right. \\
& \left.-\frac{1}{\sqrt{3}}\left(x_{2}(0)-x_{3}(0)\right) \sin \left(\frac{2 \pi t}{\tau_{\mathrm{osc}}}\right)\right\} e^{-t / \tau_{\mathrm{R}}},
\end{aligned}
$$

describing the decaying process with oscillation, with the relaxation time

$$
\tau_{\mathrm{R}}=\frac{4}{g+3},
$$

and the oscillation period

$$
\tau_{\mathrm{osc}}=\frac{8 \pi}{\sqrt{3}|p-q|},
$$

$x_{2}(t)$ and $x_{3}(t)$ can be obtained by making cyclic transformation to Eq. (27). Near equilibrium $(|p-q| \ll 1), \tau_{\text {osc }} \gg \tau_{\mathrm{R}}$, then the solution is simplified as

$$
\left\langle x_{i}(t)\right\rangle=\frac{1}{3}+\left(x_{i}(0)-\frac{1}{3}\right) e^{-t / \tau_{\mathrm{R}}},
$$

and the damped oscillation is not prominent. Fig. 5 shows the relaxation towards the uniform NESS for different $p$, with $g=-2.5$ at which only the uniform NESS is stable. By increasing $p$ from 0.5 to 1.0 , it can be seen from the direct 


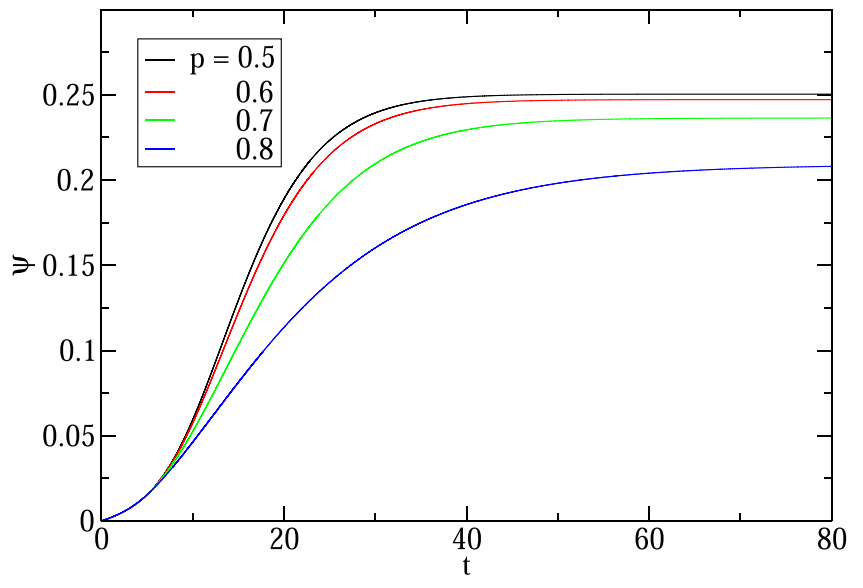

FIG. 6. Nonuniformity parameter $\psi$ as a function of $t$ for different $p$ when $g=-3.5$. The initial state is $\vec{x}(0)=\left(\frac{1}{3}, \frac{1}{3}, \frac{1}{3}\right)$ and the steady state is nonuniform. The result is numerically solved from Eq. (2) for $N=300$.

numerical calculation by Eq. (2), starting from the nonuniform initial state $\vec{x}(0)=(1,0,0)$, the relaxation time keeps almost unchanged and the oscillation in the occupation gradually appears.

When $g=-3.5$, the system stays at the nonuniform phase. To quantify the degree of nonuniformity, we define

$$
\psi \equiv \frac{1}{6}\left\langle\left(x_{1}-x_{2}\right)^{2}+\left(x_{2}-x_{3}\right)^{2}+\left(x_{3}-x_{1}\right)^{2}\right\rangle,
$$

as the "nonuniformity" parameter [27]. It is almost zero for uniform phase and becomes larger for higher nonuniformity. The relaxation towards the nonuniform NESS is illustrated by $\psi(t)$ with $g=-3.5$ in Fig. 6, showing a pure relaxation behavior. Starting from the uniform initial state $\vec{x}(0)=\left(\frac{1}{3}, \frac{1}{3}, \frac{1}{3}\right)$, the system for different $p$ all relax to the nonuniform NESS and saturates at a high nonuniformity. This can be understood in terms of the eigenvalues of the matrix $\mathbf{a}$ at the nonuniform state which are always real and negative as shown in Fig. 7.

\section{NONEQUILIBRIUM THERMODYNAMICS}

We further examine the relationship between various thermodynamical quantities, first for general nonequilibrium states and then for the NESS cases. The Boltzmann (Gibbs,

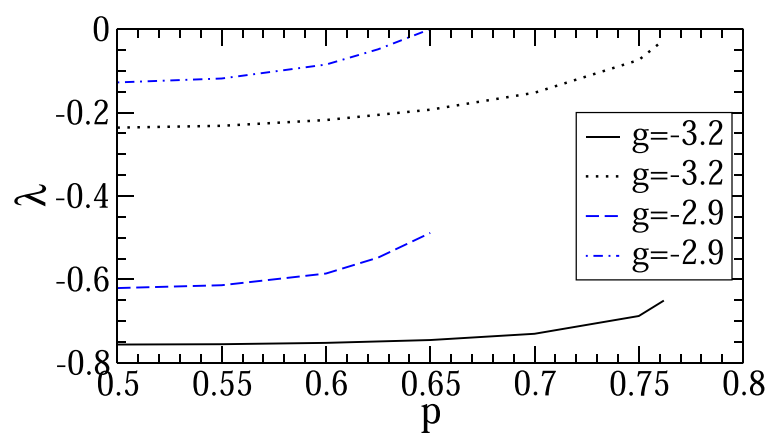

FIG. 7. The eigenvalues of a plotted against $p$, for the nonuniform NESS $(g=-3.2)$ and in the coexisting NESS $(g=-2.9)$. The relaxation to the nonuniform NESS is always purely relaxational.
Shannon) entropy of the system is given by

$$
S=-\sum_{\vec{n}} \rho(\vec{n}, t) \log \left(\rho(\vec{n}, t) / \frac{N !}{n_{1} ! n_{2} ! n_{3} !}\right),
$$

where the multiplication factor $\frac{N !}{n_{1} ! n_{2} ! n_{3} !}$ is due to the degeneracy of $\rho(\vec{n}, t)$ [2]. Applying Eq. (2), the entropy production rate above can be written as

$$
\begin{aligned}
\frac{d S}{d t}= & -\sum_{\vec{n}, \vec{m}}\left(W_{\vec{n}, \vec{m}} \rho(\vec{m}, t)-W_{\vec{m}, \vec{n}} \rho(\vec{n}, t)\right) \\
& \times \log \left(\rho(\vec{n}, t) / \frac{N !}{n_{1} ! n_{2} ! n_{3} !}\right) \\
= & \frac{N}{2} \sum_{\vec{n}, \vec{m}}\left(W_{\vec{n}, \vec{m}} \rho(\vec{m}, t)-W_{\vec{m}, \vec{n}} \rho(\vec{n}, t)\right) \\
& \times \log \left(\frac{W_{\vec{n}, \vec{m}} \rho(\vec{m}, t)}{W_{\vec{m}, \vec{n}} \rho(\vec{n}, t)}\right) \\
& +\frac{N}{2} \sum_{\vec{n}, \vec{m}}\left(W_{\vec{n}, \vec{m}} \rho(\vec{m}, t)-W_{\vec{m}, \vec{n}} \rho(\vec{n}, t)\right) \\
& \times \log \left(\frac{W_{\vec{m}, \vec{n}}}{W_{\vec{n}, \vec{m}}} \frac{N !}{\frac{N !}{n_{1} ! n_{2} ! n_{3} !}}\right) \\
= & \frac{d_{\mathrm{i}} S}{d t}+\frac{d_{e} S}{d t},
\end{aligned}
$$

where the first term is the internal entropy production rate [42], which is positive-definite and only vanishes when the system is at equilibrium [Eq. (7)]. It is the entropy produced during the irreversible process [43]. The second term refers to the entropy production rate for the reversible process [44] into the system. In the following, we will show that

$$
\frac{d_{e} S}{d t}=\beta \frac{d E}{d t}+\beta \frac{d W}{d t},
$$

where $\frac{d E}{d t}$ and $\frac{d W}{d t}$ are the rate of change of system energy and the rate of work done by the system, respectively. From the first law of thermodynamics (conservation law of energy), $T d_{e} S$ can be identified as $d Q$, the heat flow to the system from the environment. In general, during thermalization process, $d S \geqslant \beta d Q=\beta d E+\beta d W$.

Using Eqs. (3) and (4), when the particle jumps from the $i$ th to the $j$ th urn, corresponding to the transition from state $\vec{n}$ to $\vec{m}$,

$$
\frac{W_{\vec{m}, \vec{n}}}{W_{\vec{n}, \vec{m}}}=\frac{p}{q} \frac{n_{i}}{n_{j}+1} e^{\frac{g}{N}\left(n_{i}-n_{j}-1\right)},
$$

if the jump is in anticlockwise direction. Otherwise, in clockwise direction,

$$
\frac{W_{\vec{m}, \vec{n}}}{W_{\vec{n}, \vec{m}}}=\frac{q}{p} \frac{n_{i}}{n_{j}+1} e^{\frac{g}{N}\left(n_{i}-n_{j}-1\right)} .
$$

Then

$$
\begin{aligned}
\frac{d_{e} S}{d t}= & \frac{N}{2} \sum_{\vec{n}, \vec{m}}\left(W_{\vec{n}, \vec{m}} \rho(\vec{m}, t)-W_{\vec{m}, \vec{n}} \rho(\vec{n}, t)\right) \frac{g}{N}\left(n_{i}-n_{j}-1\right) \\
& +\frac{N}{2} \sum_{\vec{n}} \sum_{\vec{m}}^{\mathrm{ac}}\left(W_{\vec{n}, \vec{m}} \rho(\vec{m}, t)-W_{\vec{m}, \vec{n}} \rho(\vec{n}, t)\right) \log \left(\frac{p}{q}\right)
\end{aligned}
$$




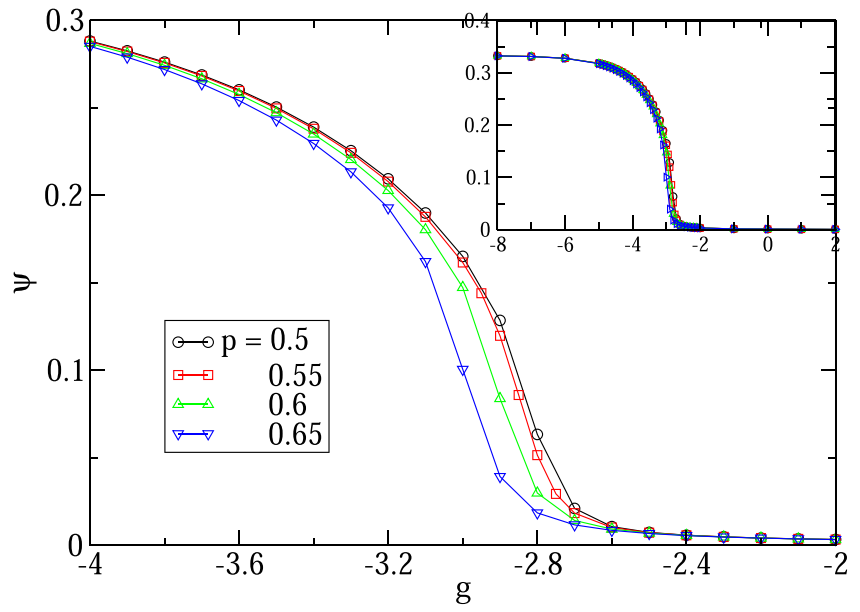

FIG. 8. Nonuniformity parameter at steady states $\psi^{s s}$ as a function of coupling constant $g$ for different $p$. Inset: The same plot with wider range of $g$. The result is obtained numerically by Eq. (40) with $N=300$.

$$
\begin{aligned}
& +\frac{N}{2} \sum_{\vec{n}} \sum_{\vec{m}}^{\mathrm{c}}\left(W_{\vec{n}, \vec{m}} \rho(\vec{m}, t)-W_{\vec{m}, \vec{n}} \rho(\vec{n}, t)\right) \log \left(\frac{q}{p}\right) \\
= & \sum_{\vec{n}, \vec{m}} g\left[n_{j}-\left(n_{i}-1\right)\right] W_{\vec{m}, \vec{n}} \rho(\vec{n}, t)-N \log \left(\frac{p}{q}\right) \\
& \times \sum_{\vec{n}} \sum_{\vec{m}}^{\mathrm{ac}}\left(W_{\vec{m}, \vec{n}} \rho(\vec{n}, t)-W_{\vec{n}, \vec{m}} \rho(\vec{m}, t)\right)
\end{aligned}
$$

where ac (c) stands for anticlockwise (clockwise) direction. The first term is the rate of change of energy $\beta \frac{d E}{d t}$ and the second term is equal to the rate of work done, which can be written as

$$
\beta \frac{d W}{d t}=-\beta \mu\left(K_{1 \rightarrow 2}+K_{2 \rightarrow 3}+K_{3 \rightarrow 1}\right),
$$

where $\mu \equiv \beta^{-1} \log \left(\frac{p}{q}\right)$ is the effective chemical potential difference to actively drive the particle from one urn to another, and the natural boundary condition is assumed. Here Eq. (34) is proved, and hence a more general thermodynamic law

$$
d S=d_{\mathrm{i}} S+\beta d E+\beta d W
$$

is derived. Note that Eqs. (38) and (39) hold even for general nonequilibrium (nonsteady state) processes.

For $p=q$, the system is at equilibrium and $\frac{d S}{d t}=\frac{d_{\mathrm{i}} S}{d t}=$ $\frac{d Q}{d t}=\frac{d E}{d t}=\frac{d W}{d t}=0$. That is, all macroscopic thermodynamic quantities do not change with time. For $p \neq q$ under NESS, since the system energy and entropy are functionals of the probability distribution and hence are time independent, thus one has $\frac{d S}{d t}=\frac{d E}{d t}=0$. Using Eqs. (38) and (39),

$$
\frac{d_{\mathrm{i}} S}{d t}=-\beta \frac{d W}{d t}=-\beta \frac{d Q}{d t}=3 K^{\mathrm{ss}} \log \left(\frac{p}{q}\right),
$$

which is a positive constant, corresponding to the housekeeping heat production rate to maintain the NESS. All the work done $(-d W)$ to the system is dissipated (measured by the internal EP $\left.d_{\mathrm{i}} S\right)$ into heat energy $(-d Q)$. Furthermore, the more nonuniform is the NESS (Fig. 8), the less is the particle flow (Fig. 3), and hence the less internal EP (Fig. 9). Since the internal EP for the nonuniform phase is always lower, the maximal EP principle could not be used to select the favorable

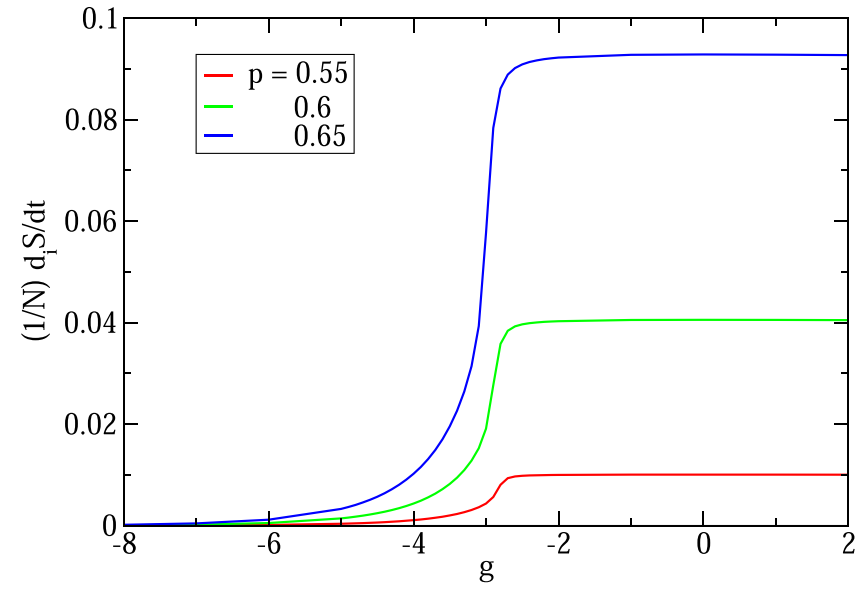

FIG. 9. Internal entropy production rate $\frac{1}{N} \frac{d_{\mathrm{i}} S}{d t}$ at steady states as a function of coupling constant $g$ for different $p$. The result is obtained numerically by Eq. (40) with $N=300$.

state in the co-existence region. The first-order nonequilibrium phase transition between the uniform and nonuniform NESS can also be observed by examining the internal EP rate as the particle attraction varies. Figure 9 shows a sharp drop near some threshold as $g$ decreases and signifying a first-order transition from the high internal EP uniform NESS to the low internal EP nonuniform NESS. Interestingly, there is a connection between the internal EP rate and the nonuniformity in the NESS. As shown in Fig. 10, when the relationship between $\frac{1}{3}-\psi^{\text {ss }}$ and $\frac{1}{N} \frac{d_{\mathrm{i}} S}{d t} /(p-q) \log \left(\frac{p}{q}\right)$ are plotted, all data with different $p$ are collapsed into a single curve. It implies the relation

$$
\left.\frac{d_{\mathrm{i}} S}{d t}\right|_{\mathrm{ss}}=N \Phi\left(\psi^{\mathrm{ss}}\right)(p-q) \log \left(\frac{p}{q}\right),
$$

where the function $\Phi\left(\psi^{\mathrm{ss}}\right)$ is some decreasing function, i.e., $\Phi^{\prime}\left(\psi^{\text {ss }}\right)<0$. To have higher internal EP rates, the system should be more uniform (lower $\psi^{\text {ss }}$ ), or with a higher direct

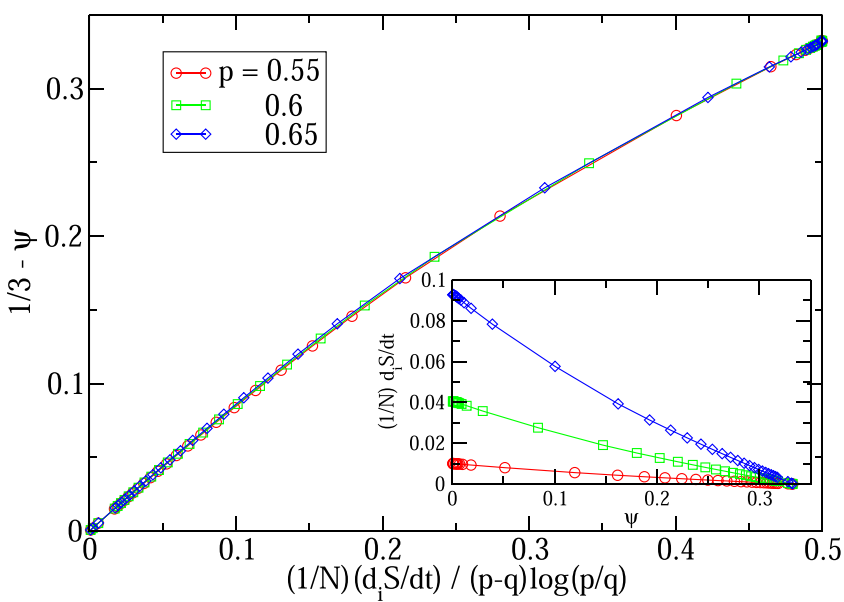

FIG. 10. The relationship between $\left(\frac{1}{3}-\psi\right)$ and $\frac{1}{N} \frac{d_{\mathrm{i}} S}{d t} \frac{1}{(p-q) \log (p / q)}$ for different $p$ at steady states. All data are collapsed into a single curve. Inset: The internal entropy production rate $\frac{1}{N} \frac{d_{\mathrm{i}} S}{d t}$ as a function of nonuniformity parameter $\psi$ for different $p$ at steady states. The result is obtained numerically with $N=300$. 
jumping rate (higher $p$ ). Figure 10 agrees with the conjecture that more uniform states have higher EP.

\section{SUMMARY AND OUTLOOK}

In this paper, we extended the Ehrenfest urn model with interactions and directional jumps, allowing for detailed investigations of the nonequilibrium steady states and associated thermodynamics. We showed that the model provides different kinds of equilibrium and nonequilibrium states. Albeit simple, the model may serve a convenient paradigm system to investigate a variety of statistical physics phenomena, ranging from equilibrium to NESS and even far from equilibrium situations.

In some situations, Landau-type free energy can be constructed for NESS near a continuous phase transition $[45,46]$ or near the saddle-point(s) of NESS states [32]. However, it is still highly nontrivial to construct or establish the existence of NESS free energy in general, especially in our case of coexisting NESS related by first-order transitions. On the other hand, because of the existence of probability density $\rho^{\mathrm{ss}}(\vec{x})$ at steady states, one may define the corresponding effective potential function $\Phi(\vec{x})=-\lim _{N \rightarrow \infty} \frac{1}{N} \log \rho^{\mathrm{ss}}(\vec{x})$. This NESS variable may reveals some NESS physical properties.

At high direct jumping rate and moderate coupling constant, the system is far from equilibrium and cannot attain the steady state but limit cycle emerges instead. If the number of urns is more than three, chaotic behavior may be possible. Such models open the possibilities of investigating systems with different degree of nonequilibrium systematically.

\section{ACKNOWLEDGMENTS}

We express our gratitude to the anonymous referees for their suggestions on the improving the readability of the manuscript. The work was supported by the Ministry of Science and Technology of the Republic of China under Grant No. 107-2112-M-008-013-MY3 and NCTS of Taiwan.

\section{APPENDIX A: STEADY-STATE SOLUTION OF MULTIVARIATE LINEAR FOKKER-PLANCK EQUATION}

The multivariate linear Fokker-Planck equation for steady state reads

$-\sum_{i} \frac{\partial}{\partial x_{i}}\left[\sum_{j} a_{i j} x_{j} \rho^{\mathrm{ss}}(\vec{x})\right]+\frac{1}{2 N} \sum_{i j} \frac{\partial^{2}}{\partial x_{i} \partial x_{j}}\left[b_{i j} \rho^{\mathrm{ss}}(\vec{x})\right]=0$,

(A1) where $\mathbf{a}$ and $\mathbf{b}$ are constant matrices of dimension $D \times D . b$ is symmetric. The natural boundary condition, $\left.\rho^{\mathrm{ss}}(\vec{x})\right|_{|\vec{x}| \rightarrow \infty}=$ 0 and $\left.\partial_{i} \rho^{\mathrm{ss}}(\vec{x})\right|_{|\vec{x}| \rightarrow \infty}=0$, is imposed. The steady state was already known [47]. In the following, we briefly outline the solution.

The form of the solution is Gaussian,

$$
\rho^{\mathrm{ss}}(\vec{x})=\left(\frac{N}{\pi}\right)^{\frac{D}{2}} \operatorname{det}(-\mathbf{c})^{\frac{1}{2}} \exp \left[N \sum_{i j} c_{i j} x_{i} x_{j}\right],
$$

where $\mathbf{c}$ is a symmetric matrix determined by $\mathbf{a}$ and $\mathbf{b}$. Substitute this form into Eq. (A1), we get two constraints,

$$
\begin{gathered}
\operatorname{tr}(\mathbf{a})=\operatorname{tr}(\mathbf{b c}), \\
x^{\mathrm{t}}(\mathbf{c a}) x=x^{\mathrm{t}}(\mathbf{c b c}) x,
\end{gathered}
$$

for any vector $x$. Equation (A3) is redundant (see below).

Notice that cbc is symmetric but ca is not necessary to be. $x^{\mathrm{t}}(\mathbf{c a}) x=x^{\mathrm{t}}\left(\mathbf{a}^{\mathrm{t}} \mathbf{c}\right) x$ since they are both numbers. Hence Eq. (A4) could be rewritten as

$$
x^{\mathrm{t}}\left(\mathbf{c a}+\mathbf{a}^{\mathrm{t}} \mathbf{c}\right) x=2 x^{\mathrm{t}}(\mathbf{c b c}) x,
$$

for any $x$, which gives

$$
\mathbf{c a}+\mathbf{a}^{t} \mathbf{c}=2 \mathbf{c b c} .
$$

Take the transpose after multiplying $\mathbf{c}^{-1}$ from the left, one can deduce Eq. (A3). Transform Eq. (A6) into

$$
\mathbf{a c} \mathbf{c}^{-1}+\mathbf{c}^{-1} \mathbf{a}^{\mathrm{t}}=2 \mathbf{b},
$$

which uniquely determine $\mathbf{c}$ by noticing that the total number of independent matrix elements of $\mathbf{c}$ is equal to the total number of independent linear equations [both are $D(D+1) / 2$ ].

The stability condition for the solution in Eq. (A2) is negative definiteness (or equivalently, the normalizability in infinite space). It is also equivalent to the fact that the odd (even) order principal minor of matrix $\mathbf{c}$ is negative (positive).

\section{APPENDIX B: DETAILED BALANCE IN MULTIVARIATE LINEAR FOKKER-PLANCK EQUATION}

If the steady state $\rho^{\mathrm{ss}}(\vec{x})$ from the multivariate linear Fokker-Planck equation in Eq. (A1) satisfies the principle of detailed balance, i.e.,

$$
\rho^{\mathrm{ss}}(\vec{x}) W\left(\vec{x}^{\prime} ; t+d t \mid \vec{x} ; t\right)=\rho^{\mathrm{ss}}\left(\vec{x}^{\prime}\right) W(\vec{x} ; t+d t \mid \vec{x} ; t),
$$

then the steady state is also called the equilibrium state. $W\left(\vec{x} ; t+d t \mid \vec{x}^{\prime} ; t\right)$ is the transition rate from one state $\vec{x}^{\prime}$ at time $t$ to another state $\vec{x}$ at time $t+d t$ [48], which can be expressed as

$$
\begin{aligned}
W\left(\vec{x}, t+d t \mid \vec{x}^{\prime}, t\right) & =\left\{1-(d t) \sum_{i j} a_{i j} x_{j}^{\prime} \frac{\partial}{\partial x_{i}}+(d t) \frac{1}{2 N} \sum_{i j} b_{i j} \frac{\partial^{2}}{\partial x_{i} \partial x_{j}}+O\left((d t)^{2}\right)\right\} \delta^{(D)}\left(\vec{x}-\vec{x}^{\prime}\right) \\
& =\exp \left[-(d t) \sum_{i j} a_{i j} x_{j}^{\prime} \frac{\partial}{\partial x_{i}}+(d t) \frac{1}{2 N} \sum_{i j} b_{i j} \frac{\partial^{2}}{\partial x_{i} \partial x_{j}}\right] \int \frac{d^{D} u}{(2 \pi)^{D}} e^{i \sum_{i} u_{i}\left(x_{i}-x_{i}^{\prime}\right)}+O\left((d t)^{2}\right) \\
& =\left(\frac{N}{2 \pi(d t)}\right)^{\frac{D}{2}}(\operatorname{det}(\mathbf{b}))^{-\frac{1}{2}} \exp \left[-\frac{N}{2(d t)} \sum_{i j}\left(\mathbf{b}^{-1}\right)_{i j}\left(x_{i}-x_{i}^{\prime}-(d t) \sum_{k} a_{i k} x_{k}^{\prime}\right)\left(x_{j}-x_{j}^{\prime}-(d t) \sum_{k} a_{j k} x_{k}^{\prime}\right)\right],
\end{aligned}
$$


and hence

$$
\begin{aligned}
\ln \left(\frac{W\left(\vec{x} ; t+d t \mid \vec{x}^{\prime} ; t\right)}{W\left(\vec{x}^{\prime} ; t+d t \mid \vec{x} ; t\right)}\right)= & \frac{N}{2} \sum_{i j}\left(\mathbf{b}^{-1}\right)_{i j} \sum_{k}\left[a_{j k}\left(x_{i}-x_{i}^{\prime}\right)\right. \\
& \left.+a_{i k}\left(x_{j}-x_{j}^{\prime}\right)\right]\left(x_{k}+x_{k}^{\prime}\right) \\
& +O(d t) .
\end{aligned}
$$

Notice that Eq. (B1) is also equivalent to

$$
\ln \left(\frac{\rho^{\mathrm{ss}}(\vec{x})}{\rho^{\mathrm{ss}}\left(\vec{x}^{\prime}\right)}\right)=\ln \left(\frac{W\left(\vec{x} ; t+d t \mid \vec{x}^{\prime} ; t\right)}{W\left(\vec{x}^{\prime} ; t+d t \mid \vec{x} ; t\right)}\right),
$$

by taking logarithm. Substitute Eq. (A2) and Eq. (B3) into Eq. (B4), and then compare the coefficients of $x_{i} x_{j}$ and that of $x_{i} x_{j}^{\prime}$ at both sides, we have

$$
\begin{gathered}
\left(\mathbf{b}^{-1} \mathbf{a}\right)_{i j}+\left(\mathbf{b}^{-1} \mathbf{a}\right)_{j i}=2 c_{i j}, \\
\left(\mathbf{b}^{-1} \mathbf{a}\right)_{i j}-\left(\mathbf{b}^{-1} \mathbf{a}\right)_{j i}=0,
\end{gathered}
$$

in which its matrix form is

$$
\mathbf{c}=\mathbf{b}^{-1} \mathbf{a} .
$$

Here we derive the linear Fokker-Planck version of detailed balance condition.

It is important to notice that if $\mathbf{c}=\mathbf{b}^{-1} \mathbf{a}$, then $\mathbf{a b}=\mathbf{b a}^{\mathrm{t}}$ by direct substitution of Eq. (B7) into Eq. (A7). If we suppose $\mathbf{a b}=\mathbf{b a}^{\mathrm{t}}$, then $\mathbf{c}=\mathbf{b}^{-1} \mathbf{a}$ is the solution of Eq. (A7). Since the solution of Eq. (A7) is unique (see Appendix A), we could draw the conclusion that Eq. (B7) holds. $\mathbf{a b}=\mathbf{b a}^{\mathrm{t}}$ is equivalent to $\mathbf{c}=\mathbf{b}^{-1} \mathbf{a}$.

Hence, $\mathbf{a b}=\mathbf{b a}^{t}$ is another equivalent statement of detailed balance of linear Fokker-Planck equation.

\section{APPENDIX C: DERIVATION FOR THE PHASE BOUNDARIES IN THE PHASE DIAGRAM}

By analyzing the stability of the saddle-points as a function of $p$ and $g$, one can obtain the phase diagram for various stable states of the three-urns model, with the phase boundary determined analytically. First by direct calculation of the matrix a at the uniform saddle point of $\left(\frac{1}{3}, \frac{1}{3}\right)$, one gets

$$
\begin{gathered}
\operatorname{tr}(\mathbf{a})=-\frac{g+3}{2}, \\
\operatorname{det}(\mathbf{a})=\frac{1}{16}\left[(g+3)^{2}+3(p-q)^{2}\right] .
\end{gathered}
$$

Hence the uniform NESS state is stable for $g>-3(\operatorname{tr}(\mathbf{a})<0$ and $\operatorname{det}(\mathbf{a})>0$ ). In addition, the eigenvalues of $\mathbf{a}$ at the uniform NESS state can be easily calculated to give

$$
\lambda=-\frac{g+3}{4} \pm i \frac{\sqrt{3}}{4}|p-q|,
$$

and hence the relaxation to the uniform NESS state always has an oscillatory component.

The behavior of the equilibrium case of $p=\frac{1}{2}$ is given in details in Ref. [27]. For the nonequilibrium case of $p \neq$ $\frac{1}{2}$, uniform, nonuniform NESS and their bistable coexisting states also occur. The phase boundary $p_{\mathrm{c}}(g)$ is determined in a similar way by the condition of saddle-node bifurcation of a pair of stable and unstable saddle-points. For a given $g, p_{\mathrm{c}}(g)$ is given by the solution of the following three equations:

$$
\begin{gathered}
A_{1}\left(x_{1}, x_{2}\right)=0, \\
A_{2}\left(x_{1}, x_{2}\right)=0, \\
\left.\frac{d x_{2}}{d x_{1}}\right|_{A_{1}=0}=\left.\frac{d x_{2}}{d x_{1}}\right|_{A_{2}=0},
\end{gathered}
$$

for the three unknowns $p_{\mathrm{c}}, x_{1}$ and $x_{2}$.

The condition of saddle-node bifurcation in Eq. (C6) can be written out explicitly as

$$
\begin{aligned}
& \frac{\frac{2 g\left[p\left(2 x_{1}+x_{2}-1\right)-x_{1}-x_{2}+1\right]}{\left(e^{g\left(2 x_{1}+x_{2}-1\right)}+1\right)^{2}}-\frac{e^{g\left(x_{1}+x_{2}\right)}\left[-g p x_{1}+g p x_{2}+g x_{1}+p+1\right]+p e^{2 g x_{2}}+e^{2 g x_{1}}}{\left(e^{g x_{1}}+e^{g x_{2}}\right)^{2}}+\frac{2 g\left[-p\left(2 x_{1}+x_{2}-1\right)+x_{1}+x_{2}-1\right]+2 p-1}{e^{g\left(2 x_{1}+x_{2}-1\right)}+1}}{\frac{g\left[-p\left(2 x_{1}+x_{2}-1\right)+x_{1}+x_{2}-1\right]}{\left(e^{g\left(2 x_{1}+x_{2}-1\right)}+1\right)^{2}}-\frac{e^{g\left(x_{1}+x_{2}\right)}\left[-g p x_{1}+g p x_{2}+g x_{1}+p\right]+p e^{2 g x_{2}}}{\left(e^{g x_{1}}+e^{g x_{2}}\right)^{2}}+\frac{g\left[p\left(2 x_{1}+x_{2}-1\right)-x_{1}-x_{2}+1\right]-p+1}{e^{g\left(2 x_{1}+x_{2}-1\right)}+1}} \\
& =\frac{\frac{(p-1) e^{2 g x_{1}}}{\left(e^{g x_{1}}+e^{g x_{2}}\right)^{2}}+\frac{e^{g\left(x_{1}+x_{2}\right)}\left[(p-1)\left(g x_{1}+1\right)-g p x_{2}\right]}{\left(e^{g x_{1}}+e^{g x_{2}}\right)^{2}}+\frac{e^{g\left(x_{1}+2 x_{2}-1\right)}\left[p\left(1-g\left(x_{1}+2 x_{2}-1\right)\right)+g x_{2}\right]+p}{\left(e^{g\left(x_{1}+2 x_{2}-1\right)}+1\right)^{2}}}{\frac{2 g\left[x_{2}-p\left(x_{1}+2 x_{2}-1\right)\right]}{\left(e^{g\left(x_{1}+2 x_{2}-1\right)}+1\right)^{2}}+\frac{2 p\left[g\left(x_{1}+2 x_{2}-1\right)-1\right]-2 g x_{2}+1}{e^{g\left(x_{1}+2 x_{2}-1\right)}+1}+\frac{e^{g\left(x_{1}+x_{2}\right)}\left\{p \sinh \left[g\left(x_{1}-x_{2}\right)\right]+(p-2) \cosh \left[g\left(x_{1}-x_{2}\right)\right]+g p x_{1}-g p x_{2}-g x_{1}+p-2\right\}}{\left(e^{g x_{1}}+e^{g x_{2}}\right)^{2}}} .
\end{aligned}
$$

The phase boundary of $p_{\mathrm{c}}(g)$ for the saddle-node bifurcation together with the $g=-3$ line for stable uniform NESS are shown in the phase diagram (Fig. 3 in main text), classifying the dynamics of the three-urns model into four regimes. In the region of $g<-3$ and $p>p_{\mathrm{c}}(g)$, there is no stable NESS state with nonsteady dynamics and the system is far from equilibrium.

Furthermore, the first-order transition line in the coexisting regime can be analytically determined as follows. The steadystate distribution near the NESS can be approximated by the Gaussian form $\exp \left(N \delta \vec{x}^{\mathrm{t}} \mathbf{c} \delta \vec{x}\right)$, where $\delta \vec{x} \equiv \vec{x}-\vec{x}^{\mathrm{sp}}$. In the coexisting regime, denote the relative weights of the uniform and nonuniform NESSs by $f(g)$ and $1-f(g)$, respectively, where the dependence on $g$ is written out explicitly. Then the steady-state distribution can be expressed as

$$
\rho^{\mathrm{ss}}(\vec{x})=\mathcal{N}^{-1}\left(f(g) e^{N \delta x_{\mathrm{u}}^{\vec{t}} \mathbf{c}_{\mathrm{u}} \delta \vec{x}_{u}}+[1-f(g)] e^{N \delta \vec{x}_{\mathrm{nu}}^{t} \mathbf{c}_{\mathrm{mu}} \delta \vec{x}_{\mathrm{nu}}}\right),
$$

where

$$
\mathcal{N}=\int d^{2} x\left(f(g) e^{N \delta \vec{x}_{\mathrm{u}}^{t} \mathbf{c}_{\mathrm{u}} \delta \vec{x}_{\mathrm{u}}}+[1-f(g)] e^{N \delta \vec{x}_{\mathrm{mu}}^{t} \mathbf{c}_{\mathrm{mu}} \delta \vec{x}_{\mathrm{nu}}}\right)
$$

is the normalization factor, and the subscripts $\mathrm{u}$ and nu denote uniform and nonuniform NESS, respectively. The ensemble 


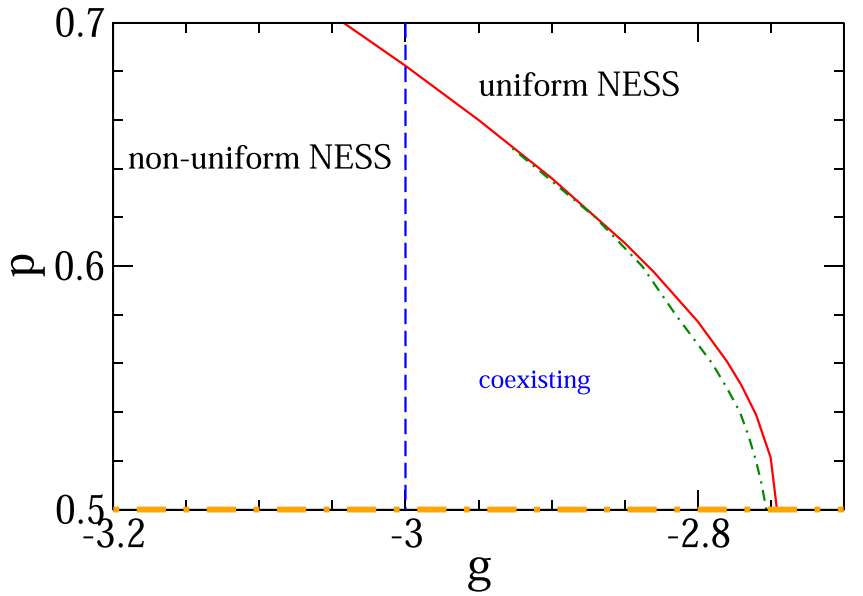

FIG. 11. Close-up view of the phase diagram in Fig. 4 of the interacting model of three urn model near the coexistence regime. The dashed-dotted curve denotes the first-order transition line.

average of the steady-state flux can be computed using saddle point approximation to give

$$
\left\langle K^{\mathrm{ss}}(g)\right\rangle \simeq \chi(g) K_{\mathrm{u}}^{\mathrm{ss}}+[1-\chi(g)] K_{\mathrm{nu}}^{\mathrm{ss}}(g),
$$

where

$$
\chi(g)=\frac{f(g)}{f(g)+[1-f(g)] \sqrt{\frac{\operatorname{det}\left(\mathbf{c}_{u}(g)\right)}{\operatorname{det}\left(\mathbf{u}_{\mathrm{nu}}(g)\right)}}} .
$$

At the first-order transition point $g_{\mathrm{t}}, f\left(g_{\mathrm{t}}\right)=\frac{1}{2}$ and the righthand side of Eq. (C10) reduces to

$$
\phi(g) K_{\mathrm{u}}^{\mathrm{ss}}+[1-\phi(g)] K_{\mathrm{nu}}^{\mathrm{ss}}(g),
$$

where

$$
\phi(g)=\frac{1}{1+\sqrt{\frac{\operatorname{det}\left(\mathbf{c}_{\mathrm{u}}(g)\right)}{\operatorname{det}\left(\mathbf{c}_{\mathrm{nu}}(g)\right)}},}
$$

which can be analytically calculated as a function of $g$. Thus by numerically computing $\left\langle K^{\text {ss }}(g)\right\rangle$ using the numerical solution of Eq. (2), $g_{\mathrm{t}}$ can be obtained from the intersection of the curves of $\left\langle K^{\mathrm{ss}}(g)\right\rangle$ and Eq. (C12). For given values of $p$ in the coexistence regime, $g_{\mathrm{t}}$ is obtained theoretically from the above manner and the result of the first-order transition line is shown in Fig. 11. The first-order line is rather close to the stability boundary of the nonuniform NESS indicates that the nonuniform NESS dominates over the uniform NESS in the coexistence regime. This echoes with the observation in Fig. 7 that the eigenvalues of a of the nonuniform NESS are much more negative than that of (the real part) the uniform NESS unless $p$ is very close to the stability boundary, indicating that the nonuniform NESS is a strong attractor than that of the uniform NESS in most of the coexistence regime.
[1] P. Ehrenfest and T. Ehrenfest, Uber zwei bekannte Einwande gegen das Boltzmannsche H-Theorem, Phys. Z. 8, 311 (1907).

[2] K. Huang, Statistical Mechanics, 2nd ed. (Wiley, New York, 1987).

[3] H. Poincare, Sur le problème des trois corps et les èquations de la dynamique, Acta Math. 13, 1 (1890).

[4] M. Kac, Random walk and the theory of Brownian motion, Am. Math. Mon. 54, 369 (1947).

[5] A. J. F. Siegert, On the approach to statistical equilibrium, Phys. Rev. 76, 1708 (1949).

[6] M. J. Klein, Generalization of the Ehrenfest urn model, Phys. Rev. 103, 17 (1956).

[7] Y. M. Kao and P. G. Luan, Poincare cycle of a multibox Ehrenfest urn model with directed transport, Phys. Rev. E 67, 031101 (2003).

[8] J. Nagler, Directed and undirected multiurn models in a onedimensional ring, Phys. Rev. E 72, 056129 (2005).

[9] J. Clark, M. Kiwi, F. Torres, J. Rogan, and J. A. Valdivia, Generalization of the Ehrenfest urn model to a complex network, Phys. Rev. E 92, 012103 (2015).

[10] J. Nagler, C. Hauert, and H. G. Schuster, Self-organized criticality in a nutshell, Phys. Rev. E 60, 2706 (1999).

[11] J. P. Bouchaud and A. Georges, Anomalous diffusion in disordered media: Statistical mechanics, models and physical applications, Phys. Rep. 195, 127 (1990).

[12] J. P. Boon and J. F. Lutsko, Nonlinear diffusion from Einstein's master equation, Europhys. Lett. 80, 60006 (2007).
[13] J. F. Lutsko and J. P. Boon, Microscopic theory of anomalous diffusion based on particle interactions, Phys. Rev. E 88, 022108 (2013).

[14] V. Schwammle, F. D. Nobre, and E. M. F. Curado, Consequences of the $\mathrm{H}$ theorem from nonlinear Fokker-Planck equations, Phys. Rev. E 76, 041123 (2007).

[15] M. Shiino, Free energies based on generalized entropies and Htheorems for nonlinear Fokker-Planck equations, J. Math. Phys. 42, 2540 (2001).

[16] T. D. Frank and A. Daffertshofer, H-theorem for nonlinear Fokker-Planck equations related to generalized thermostatistics, Phys. A 295, 455 (2001).

[17] P. H. Chavanis, Generalized thermodynamics and FokkerPlanck equations: Applications to stellar dynamics and twodimensional turbulence, Phys. Rev. E 68, 036108 (2003).

[18] A. Lipowski and M. Droz, Urn model of separation of sand, Phys. Rev. E 65, 031307 (2002).

[19] A. Lipowski and M. Droz, Moment ratios for an urn model of sand compartmentalization, Phys. Rev. E 66, 016118 (2002).

[20] F. Coppex, M. Droz, and A. Lipowski, Dynamics of the breakdown of granular clusters, Phys. Rev. E 66, 011305 (2002).

[21] G. M. Shim, B. Y. Park, and H. Lee, Analytic study of the urn model for separation of sand, Phys. Rev. E 67, 011301 (2003).

[22] G. A. Casas, F. D. Nobre, and E. M. F. Curado, Nonlinear Ehrenfest's urn model, Phys. Rev. E 91, 042139 (2015).

[23] E. M. F. Curado and F. D. Nobre, Derivation of nonlinear Fokker-Planck equations by means of approximations to the master equation, Phys. Rev. E 67, 021107 (2003). 
[24] F. D. Nobre, E. M. F. Curado, and G. Rowlands, A procedure for obtaining general nonlinear Fokker-Planck equations, Phys. A 334, 109 (2004).

[25] T. D. Frank, Nonlinear Fokker-Planck Equations (Springer, New York, 2005).

[26] C. H. Tseng, Y. M. Kao, and C. H. Cheng, Ehrenfest urn model with interaction, Phys. Rev. E 96, 032125 (2017).

[27] C. H. Cheng, B. Gemao, and P. Y. Lai, Phase transitions in Ehrenfest urn model with interactions: Coexistence of uniform and nonuniform states, Phys. Rev. E 101, 012123 (2020).

[28] G. Nicolis and I. Prigogine, Self Organization in Nonequilibrium Systems (Wiley, New York, 1977).

[29] Entropy Measures, Maximum Entropy Principle and Emerging Applications, edited by J. Kameshu and N. R. Pal (Springer, New Delhi, 2003).

[30] W. T. Grandy Jr., Entropy and the Time Evolution of Macroscopic Systems (Oxford University Press, Oxford, 2008).

[31] In equilibrium statistical mechanics, when $N$ is large, in computing the partition function or any average physical variables, the method of steepest decent (one of the popular asymptotic methods) is applied. In this way, the physical variables we consider will be extended from real to complex plane. The "optimal" point in this complex plane is not locally maximum or minimum, but a saddle point (Chap. 9 in Ref. [2]). This saddle point in the complex plane corresponds to the fixed point in the parameter space of the Fokker-Planck Equation in Eq. (14).

[32] C. Kwon, P. Ao, and D. Thouless, Structure of stochastic dynamics near fixed points, Proc. Natl. Acad. Sci. USA 102, 13029 (2005).

[33] K.-H. Chiang, C.-L. Lee, P.-Y. Lai, and Y.-F. Chen, Electrical autonomous Brownian gyrator, Phys. Rev. E 96, 032123 (2017).

[34] H. Chang, C. L. Lee, P. Y. Lai, and Y. F. Chen, Autonomous Brownian gyrators: A study on gyrating characteristics, Phys. Rev. E 103, 022128 (2021).
[35] H. A. Kramers, Brownian motion in a field of force and the diffusion model of chemical reactions, Physica 7, 284 (1940).

[36] J. E. Moyal, Stochastic processes and statistical physics, J. R. Stat. Soc. Ser. B 11, 150 (1949).

[37] The WKB method is adopted in Section 6.6.7 from Ref. [48], which is the usual analytical method to solve the Fokker-Planck equation with small diffusion coefficient (large $N$ limit in our case).

[38] R. Kubo, K. Matsuo, and K. Kitahara, Fluctuation and relaxation of macrovariables, J. Stat. Phys. 9, 51 (1973).

[39] M. I. Dykman, E. Mori, J. Ross, and P. M. Hunt, Large fluctuations and optimal paths in chemical kinetics, J. Chem. Phys. 100, 5735 (1994).

[40] V. Elgartv and A. Kamenev, Rare event statistics in reactiondiffusion systems, Phys. Rev. E 70, 041106 (2004).

[41] M. Assaf and B. Meerson, WKB theory of large deviations in stochastic populations, J. Phys. A 50, 263001 (2017).

[42] J. Schnakenberg, Network theory of microscopic and macroscopic behavior of master equation systems, Rev. Mod. Phys. 48, 571 (1976).

[43] I. Prigogine, Non-Equilibrium Statistical Mechanics (Dover, New York, 2017).

[44] G. Lebon, D. Jou, and J. Casas-Vazquez, Understanding Non-equilibrium Thermodynamics. Foundations, Applications, Frontiers (Springer, Berlin, 2007).

[45] H. Haken, Information and Self-Organization, A Macroscopic Approach to Complex Systems (Springer, New York, 1988).

[46] C. Aron and C. Chamon, Landau theory for nonequilibrium steady states, SciPost Phys. 8, 074 (2020).

[47] M. C. Wang and G. E. Uhlenbeck, On the Theory of the Brownian Motion II, Rev. Mod. Phys. 17, 323 (1945).

[48] H. Risken, The Fokker-Planck Equation (Springer, New York, 1983). 